\title{
Peripheral and Central Auditory Manifestation of Rheumatoid Arthritis
}

\author{
Sanjay Kumar Munjal ${ }^{1 *}$, Parul Sud ${ }^{1}$, Sunita Gudwani ${ }^{2}$, Aman Sharma ${ }^{1}$, Anuradha Sharma ${ }^{1}$ and Naresh K \\ Panda $^{1}$ \\ ${ }^{1}$ Post Graduate Institute of Medical Education and Research, India \\ ${ }^{2}$ All India Institute of Medical Education, India
}

Submission: December 14, 2016; Published: January 05, 2017

*Corresponding author: Sanjay Kumar Munjal, Speech and Hearing, Department of Otolaryngology, Head and Neck Surgery, Post Graduate Institute of Medical Educational and Research, Chandigarh, 160012, INDIA. Tel: +91-01722756955; Fax: +91-172-2744401, +91-172-2745078; Email: sanjaymunjal1@hotmail.com

\begin{abstract}
The present study was conducted with the aim to investigate the peripheral and central auditory manifestations in individuals with RA. 40 subjects (age range: 25-45 years), diagnosed with RA and 40 age and sex matched otologically normal subjects participated in the study. Participants underwent a comprehensive audiological test battery comprising of conventional and extended high frequency puretone audiometry, impedance audiometry, otoacoustic emissions (OAEs) and auditory brainstem responses (ABR). Significant difference between the puretone thresholds for the two groups was found at high frequencies (beyond $4 \mathrm{kHz}$ ). Majority of the subjects in RA group had absent/reduced amplitude for TEOAE. A highly statistically significant difference was observed between the RA and the control group for the absolute latency of wave I, III and V of the left ear. Considering the high incidence of hearing loss in RA group, a regular referral for audiological evaluations is suggested.

Keywords: Rheumatoid arthritis, Sensori neural hearing loss, Acoustic stapedial reflex, Auditory brainstem response, Oto acoustic emissions, High frequency audiometry

Abbreviations: RA: Rheumatoid Arthiritis; SNHL: Sensory-Neural Hearing Loss; HL: Hearing Loss; OAE: Oto Acoustic Emissions; ABR: Auditory Brainstem Response; PTA: Pure Tone Audiometry; CHL: Conductive Hearing Loss; ART: Acoustic Reflex Threshold; SNR: Signal to Noise Ratio; IHS: Intelligent Hearing System; IPL: InterPeak Latency; TEOAE: Transient Evoked Oto Acoustic Emissions; ESR; Erythrocyte Sedimentation Rate; RF: Rheumatoid Factor; ANA: Antinuclear Antibody
\end{abstract}

\section{Introduction}

Rheumatoid arthritis (RA) is a chronic multisystem disease. It is associated with a number of systemic manifestations. RA is a common autoimmune disease affecting $2 \%$ to $3 \%$ of the adult population [1]. The usual age of onset is from 35 to 45 years [2]. The characteristic trait of RA is persistent inflammatory synovitis, which involves the peripheral joints in a symmetric distribution. The hallmark characteristic of the disease is the potential of the synovial inflammation to cause cartilage damage, bone erosions and subsequent changes in joint integrity [3]. RA has the potential to affect the form and function of many otolaryngological joints, such as the ossicular joints located in the ear, the temporomandibular joint, and the cricoarytenoid joints [4-6]. The incudomalleolar and incudostapedial joints of the middle ear are freely movable joints called diathroses.

These joints may be subject to rheumatic involvement. Similar to ossicular joints, the cricoarytenoid joint is a true diathrodial joint and a high occurrence of rheumatoid laryngitis has been documented [7-10]. Despite the prevalence of otolaryngologic complications of RA, the manifestations of the disease cause auditory symptoms that are often ignored by both patient and physician [9]. Sensory-neural hearing loss (SNHL) involving the cochlear mechanism is a common finding in patients with RA [11-14]. Conductive hearing loss [13,15] and mixed hearing loss (HL) $[14,15]$ have also been reported among the target population. SNHL may be the result of the extraarticular manifestation of the disease (rheumatoid nodular vasculitis) or due to drug ototoxicity [16-18]. Increased laxity of the middle ear transducer mechanism (because of the synovial joints between the ossicles in the middle ear) is likely the cause of conductive element. Also, the presence of a mixed hearing loss suggests a multifocal involvement of the auditory system in RA [13].

A brief review of the literature on RA presents with conflicting results suggesting hearing loss to be conductive, SN, 
or mixed type. The affected frequencies are low $(0.125 \mathrm{kHz}-$ $500 \mathrm{~Hz})$ or high $(8 \mathrm{kHz})$ and the pathophysiology of the disease could be in the ossicular chain or cochlea, depending on the type of hearing loss and disease progression. These inconsistent findings necessitated us to investigate and profile audiological characteristics of the subjects with RA. The main objective of the study was to investigate the peripheral and central auditory manifestations in individuals with RA. These were compared with the control group (normal hearing population). The final objective of the study was to correlate the audiological changes in RA with other blood investigations to test the active disease progression.

\section{Materials and Methods}

A comparative case-control study was carried out at the Speech and Hearing Unit, Department of ENT, Post Graduate Institute of Medical Education and Research, Chandigarh. 40 subjects in the age range of 25 to 45 years with a mean age of 34.5 years, diagnosed with RA (Group 1) and 40 age and sex matched subjects with no history of any previous otological or audiological complaint (Group 2) were enrolled in the present study. Subjects with a history of ear disease (tympanic membrane perforation, otitis media, otosclerosis, otorrhoea), inner ear disease (Meniere's disease), severe head injury, history of previous otologic surgery, metabolic or systemic diseases associated with hearing loss, loud noise exposure of drug ototoxicity, individuals with family history of hearing loss were excluded from the present study. An informed consent was taken from all the participants and the study was approved by the institute ethical committee.

All the participants underwent a comprehensive audiological test battery comprising of initial otoscopic examination to check the status of the tympanic membrane and the external ear canal, conventional and extended high frequency puretone audiometry with bone conduction audiometry, impedance audiometry with stapedial reflex testing, otoacoustic emissions (OAEs) and auditory brainstem responses (ABR). Pure tone clinical audiometer (Madsen Orbiter 922, Version 2; Madsen Electronics, Taastrup, Denmark) was used for performing conventional audiometry $(250 \mathrm{~Hz}-8000 \mathrm{~Hz})$ and extended high frequency audiometry $(10,000 \mathrm{~Hz}-20,000 \mathrm{~Hz})$. Puretone audiometry (PTA) was conducted with TDH 39 supra-aural earphones(Telephonics Co., Farmingdale, USA) with noise excluding headsets and extended high frequency puretone audiometry was performed using HDA200 closed circumaural earphones(Sennheiser Co., Germany).

Bone conduction hearing thresholds were estimated using bone vibrator Radio ear B71 with head band. The air bone gap obtained with air conduction and bone conduction thresholds confirmed the type of hearing loss as sensorineural (SN) or conductive hearing loss (CHL). Hearing loss were graded according to the recommendation by American Speech and Hearing Association, (ASHA) [19], as minimal, mild, moderate, moderately severe, severe and profound. Pure tone Average (PTA1, PTA2, and PTA3) were calculated for all test frequencies. PTA 1 was computed as an average of $0.5 \mathrm{KHz}, 1 \mathrm{~K} \mathrm{~Hz}$, and $2 \mathrm{KHz}$; PTA 2 of $4 \mathrm{KHz}, 8 \mathrm{KHz}$ and $10 \mathrm{KHz}$ and PTA 3 of $12 \mathrm{KHz}, 14 \mathrm{KHz}$, $16 \mathrm{KHz}$, and $20 \mathrm{KHz}$.

Immitance audiometry and Stapedial Reflex testing were conducted using a commercially available impedance meter (Maico MI34 Middle ear analyzer, MAICO Diagnostics, Berlin) calibratled as per ANSI 1992 standards. The test protocol included a low frequency probe tone $(226 \mathrm{~Hz})$ with pressure variations from -400 to 200 dapa. Tympanogram obtained were classified according to Jerger and Jerger classification system [20]. Acoustic reflex threshold (ART) was measured for both ipsilateral and contralateral ears at $500 \mathrm{~Hz}, 1 \mathrm{kHz}, 2 \mathrm{kHz}$ and $4 \mathrm{kHz}$ Ipsilateral ART between 70-90 dB HL was considered as present and, between 95-110 dB were considered as elevated and above $110 \mathrm{~dB}$ HL as absent. Contralateral ART between 80$100 \mathrm{~dB}$ HL was considered as present and between 105-120 dB HL as elevated and above $120 \mathrm{~dB}$ HL as absent.

Otoacoustic emissions (OAEs) were measured using the Smart TrOAE and Smart OAE(Intelligent Hearing System, Miami, Florida, USA). The amplitude and signal to noise ratio (SNR) at frequencies $1,000 \mathrm{~Hz}, 1500 \mathrm{~Hz}, 2,000,3,000 \mathrm{~Hz}$ and 4,000 $\mathrm{Hz}$ were used to interpret the test results. The SNR of $>3 \mathrm{~dB}$ at three consecutive frequencies was considered as presence of response. Electrophysiological tests to detect the site of the lesion along the auditory pathway (auditory nerve and lower auditory brainstem) were also a part of the test battery. Auditory Brainstem Responses (ABR) recording was done using NeuroAudio (Version 2010), (Neurosoft, Ivanovo, Russia). Electrode placement was as per 10-20 international systems (M1CzM2Fz,) with impedance measurements below $5 \mathrm{~K} \Omega$. Silver- silver chloride button electrodes were used for testing [19].

ABR was performed using filtered clicks at a rate of $19.3 / \mathrm{sec}$, with filters setting between $100 \mathrm{~Hz}$ to 3,000 Hz. Every response was an average of 1,024 presentations. Monaural recordings were performed at $90 \mathrm{~dB} \mathrm{nHL}$ (hearing level for normal individuals) and $70 \mathrm{~dB}$ nHL for both ears. The absolute latencies of wave I, III \& V and interpeak latencies (IPL) of Wave I-III, III-V and I-V were evaluated. Reproducibility of the waveform was checked by presenting the stimulus twice. ABR findings were reported abnormal for IPL delay of waves I to III, $>2.4 \mathrm{msec}$; waves III to V, $>2.2 \mathrm{msec}$; and waves I to $\mathrm{V},>4.4 \mathrm{msec}$.

\section{Results}

\section{Pure tone audiometry}

$32(80 \%)$ out of 40 subjects in the RA group were found to have hearing loss in right ear and 28 (70\%) subjects had hearing loss in left ear, based on PTA1. Based on PTA2, 37(92.5\%) and $36(90 \%)$ subject had hearing loss in right and left ear respectively. All the subjects (100\%) in the RA group had hearing loss at PTA3 for both ears. The results of PTA1 revealed that 24 


\section{Global Journal of Otolaryngology}

out of 32 had a mild degree of hearing loss in right ear and 18 out of 28 had a mild degree of hearing loss in left ear. A moderate degree of hearing loss was present in 5 and 8 subjects in right and left ear respectively. 3 subjects had severe degree of hearing loss in right ear and 2 had hearing loss in left ear.

The majority of the subjects had SN hearing loss (92.5\%) except three cases (7.5\%) that reported CHL, based on the bone conduction thresholds and air bone gap. Statistical analysis was carried out to compare the pure tone thresholds between the RA and control group using Student's t- test. (Figure 1 and Table 1) show the comparison between control and the RA Group on PTA. Results revealed a statistically significant $(p<0.001)$ difference between the two groups for all the conventional as well as extended high frequencies.

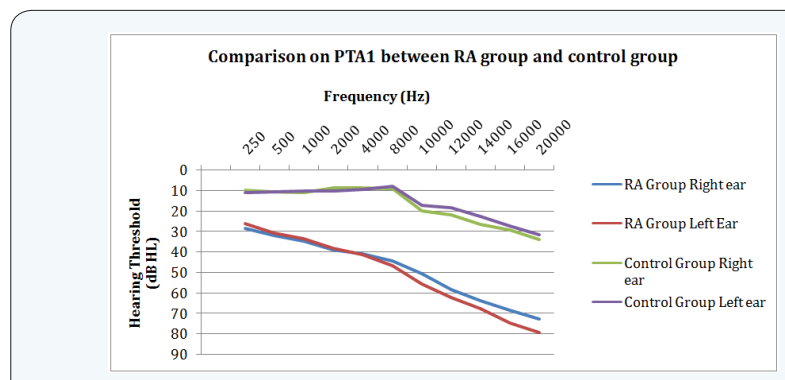

Figure 1: Comparison between the RA group and control group on Pure Tone Audiometry.

Table 1: Comparison between RA and control group for pure tone audiometry ( ${ }^{* *} p<0.001$ ).

\begin{tabular}{|c|c|c|c|c|c|c|}
\hline \multirow{2}{*}{$\begin{array}{c}\text { Frequency } \\
\text { (Hz) }\end{array}$} & \multicolumn{3}{|c|}{ RA Group } & \multicolumn{3}{|c|}{ Control Group } \\
\hline & Ear & $\begin{array}{c}\text { Mean } \\
\text { (dBHL) }\end{array}$ & S.D & $\begin{array}{c}\text { Mean } \\
\text { (dBHL) }\end{array}$ & S D & t-value \\
\hline \multirow{2}{*}{250} & Right & 28.25 & 11.354 & 10.00 & 3.922 & $9.609^{* * *}$ \\
\hline & Left & 25.88 & 15.059 & 10.75 & 6.655 & $5.810^{* * *}$ \\
\hline \multirow{2}{*}{500} & Right & 31.62 & 10.463 & 10.50 & 4.356 & $11.789 * * *$ \\
\hline & Left & 30.50 & 11.810 & 10.50 & 4.777 & $9.929 * * *$ \\
\hline \multirow{2}{*}{1000} & Right & 34.50 & 13.858 & 11.25 & 4.494 & $10.093^{* * *}$ \\
\hline & Left & 33.25 & 15.298 & 10.25 & 5.057 & $9.028^{* * *}$ \\
\hline \multirow{2}{*}{2000} & Right & 38.88 & 20.048 & 8.75 & 6.770 & $9.004^{* * *}$ \\
\hline & Left & 38.00 & 17.570 & 10.12 & 6.252 & $9.453^{* * *}$ \\
\hline \multirow{2}{*}{ PTA1 } & Right & 35.00 & 14.157 & 10.16 & 3.936 & $10.688^{* * *}$ \\
\hline & Left & 33.92 & 14.620 & 10.29 & 4.235 & $9.816^{* * *}$ \\
\hline \multirow{2}{*}{4000} & Right & 40.75 & 19.200 & 8.88 & 5.487 & $10.095^{* * *}$ \\
\hline & Left & 41.12 & 21.438 & 9.25 & 6.460 & $9.004^{* * *}$ \\
\hline \multirow{2}{*}{8000} & Right & 44.12 & 19.771 & 9.00 & 7.861 & $10.441^{* * *}$ \\
\hline & Left & 46.38 & 20.908 & 7.75 & 8.393 & $10.843^{* * *}$ \\
\hline \multirow{2}{*}{10000} & Right & 50.25 & 20.222 & 19.75 & 5.424 & $9.214^{* * *}$ \\
\hline & Left & 55.25 & 20.411 & 17.12 & 3.180 & $11.673^{* * *}$ \\
\hline \multirow{2}{*}{ PTA2 } & Right & 45.04 & 18.259 & 12.54 & 4.575 & $10.312^{* * *}$ \\
\hline & Left & 46.01 & 18.223 & 12.55 & 4.580 & $10.919^{* * *}$ \\
\hline \multirow{2}{*}{12000} & Right & 58.12 & 21.652 & 21.75 & 5.377 & $10.312^{* * *}$ \\
\hline & Left & 62.12 & 23.829 & 18.38 & 2.628 & $11.542^{* * *}$ \\
\hline \multirow{2}{*}{14000} & Right & 63.75 & 23.825 & 26.38 & 4.081 & $9.779^{* * *}$ \\
\hline & Left & 67.38 & 22.475 & 22.50 & 2.774 & $12.533^{* * *}$ \\
\hline \multirow{2}{*}{16000} & Right & 68.12 & 25.082 & 29.25 & 3.499 & $9.709 * * *$ \\
\hline & Left & 74.38 & 25.349 & 27.25 & 2.519 & $11.700^{* * *}$ \\
\hline \multirow{2}{*}{20000} & Right & 72.50 & 27.410 & 33.75 & 3.349 & $8.875^{* * *}$ \\
\hline & Left & 79.00 & 23.837 & 31.62 & 2.372 & $12.508^{* * *}$ \\
\hline \multirow{2}{*}{ PTA3 } & Right & 65.62 & 23.952 & 27.78 & 3.379 & $9.895^{* * *}$ \\
\hline & Left & 70.72 & 22.988 & 24.94 & 1.833 & $12.556^{* * *}$ \\
\hline
\end{tabular}


Global Journal of Otolaryngology

Table 2: Comparison between RA Group and Control Group on Auditory Brainstem Response testing.

\begin{tabular}{|c|c|c|c|c|c|c|}
\hline \multirow{2}{*}{ Parameter } & \multicolumn{3}{|c|}{ RA Group } & \multicolumn{3}{|c|}{ Control Group } \\
\hline & Ear & $\operatorname{Mean}(\mathrm{ms})$ & S.D & Mean(ms) & S D & 't'value \\
\hline \multirow{2}{*}{ Wave I } & Right & 1.57 & 0.234 & 1.55 & 0.060 & 0.601 \\
\hline & Left & 1.64 & 0.219 & 1.53 & 0.064 & $3.104^{* *}$ \\
\hline \multirow{2}{*}{ Wave III } & Right & 3.75 & 0.207 & 3.70 & 0.148 & 1.166 \\
\hline & Left & 3.79 & 0.184 & 3.66 & 0.145 & $3.387^{* * *}$ \\
\hline \multirow{2}{*}{ Wave V } & Right & 5.61 & 0.182 & 5.54 & 0.132 & 1.821 \\
\hline & Left & 5.62 & 0.155 & 5.55 & 0.160 & $2.092^{*}$ \\
\hline \multirow{2}{*}{ Wave I-III } & Right & 2.24 & 0.361 & 2.15 & 0.151 & 1.450 \\
\hline & Left & 2.19 & 0.236 & 2.13 & 0.135 & 1.559 \\
\hline \multirow{2}{*}{ Wave III-V } & Right & 1.88 & 0.124 & 1.84 & 0.164 & 1.170 \\
\hline & Left & 1.83 & 0.132 & 1.88 & 0.119 & 1.743 \\
\hline \multirow{2}{*}{ Wave I-V } & Right & 4.02 & 0.457 & 3.99 & 0.124 & 0.280 \\
\hline & Left & 4.01 & 0.245 & 4.01 & 0.153 & 0.109 \\
\hline
\end{tabular}

${ }^{*} p<0.05 ;{ }^{* *} p<0.01 ;{ }^{* * *} p<0.001$

\section{Tympanometry}

Normal functioning middle ears revealed by Type A tympanograms were obtained for 36 (90\%) and 37 (92.5\%) for the right and left ear respectively. Similar findings were obtained for all subjects in the control group with a Type A tympanogram suggestive of normal middle ear statuses. Type B tympanogram was present in three subjects suggesting middle ear pathology and one subject had type $C$ tympanogram in both ears indicative of negative middle ear pressure/ Eustachian tube dysfunction.

\section{Stapedial Reflex Test}

Ipsilateral acoustic reflexes were absent in 31(77.5\%) subjects and present in $9(22.5 \%)$ subjects with RA. Contralateral acoustic reflexes were absent in 35(87.5\%) and present in $5(12.5 \%)$ subjects with RA. For the control group, ipsilateral and contralateral reflexes were absent in $3(7.5 \%)$ subjects.

\section{Otoacoustic Emissions}

In the RA group, TEOAE were absent/reduced amplitude in $29(72.5 \%)$ subjects in right ear and 27(67.5\%) subjects in left ear. No subjects in the control group had absent TEOAE [20-24].

\section{Auditory Brainstem Responses}

In the RA group wave I-III interpeak latency delay was seen in $7(17.5 \%)$ subjects and $4(10 \%)$ subjects in right and left ear respectively. In the control group, none of the subjects had wave I-III interpeak latency delay. Wave III-V interpeak latency delay was not observed in any of the subjects in both study and control groups. However, Wave I-V interpeak latency delay was present in $3(7.5 \%)$ and $2(5 \%)$ subjects of the study group in right and left ear respectively. None of the subjects in the control group had Wave I-V interpeak latency delay. Comparisons of the absolute latencies and IPL difference for the Wave I, III and V for both groups were performed using the Student's t-test. Table 2 shows the comparison of absolute latencies and IPL difference on ABR testing between RA and control group using Independent Student's t-test.

A highly statistically significant difference was observed between the RA and control group for the absolute latency of wave I, III $\left(\mathrm{p}<0.01 ;{ }^{* * *} \mathrm{p}<0.001\right)$ and Wave V(*p $\left.<0.05\right)$ of the left ear. However, no significant difference was seen between the two groups for interpeak latencies of I-III, III-V, and I-V. The results of blood investigations (Erythrocyte Sedimentation Rate (ESR)/Rheumatoid Factor (RF)/Antinuclear Antibody (ANA) were correlated with the audiological parameters using the Pearson correlation test. No statistically significant correlation was observed between ESR, ANA and any of the audiological parameters investigated. A significant correlation existed between the Rheumatoid factor (RF) and puretone thresholds. The results of the Pearson correlation test for RF and puretone thresholds have been presented in (Table 3).

Table 3: Correlation between Rheumatoid factor and Pure tone thresholds.

\begin{tabular}{|c|c|c|c|c|c|c|}
\hline RF & \multicolumn{2}{|c|}{ PTA1 } & \multicolumn{2}{c|}{ PTA2 } & \multicolumn{2}{c|}{ PTA3 } \\
\hline EAR & RIGHT & LEFT & RIGHT & RIGHT & 0.427 & 0.441 \\
\hline $\begin{array}{c}\text { Correlation } \\
\text { Coefficient(r) }\end{array}$ & 0.571 & 0.551 & 0.600 & 0.557 & .001 & .01 \\
\hline p value & .001 & .001 & .001 & .001 & .01 \\
\hline
\end{tabular}

RA: Rheumatoid Arthiritis; SD: Standard Deviation; PTA: Puretone Audiometry; RF: Rheumtoid Factor 


\section{Global Journal of Otolaryngology}

\section{Discussion}

There is much inconclusive evidence that supports the involvement of peripheral or central auditory structures in rheumatoid arthritis. Several authors report a middle ear involvement associated with disease progression in rheumatoid arthritis [13,15,25-27], others suggest a sensory/ neural component related to hearing loss[11,12,15,16]. The confounding results lead us to carry out the present study with an aim to profile the audiological status of subjects with RA and compare the findings with the control group. The results of the present study reveal $80 \%$ and $70 \%$ subjects had hearing loss in the right and left ear respectively for the PTA1 (Average of $0.5 \mathrm{kHz}, 1 \mathrm{kHz}$, and $2 \mathrm{kHz}$ ). The present study also revealed a significant high frequency hearing loss in both ears for the study group (100\% subjects with hearing loss in both ears).

Several authors $[13,17,21,22,28,29]$ have also reported similar findings wherein individuals with RA reported a higher degree of hearing loss compared to their age and gender matched controls. Treviño et al. [30] reported a high prevalence of sensory neural hearing loss in high and ultra high frequencies for subjects with rheumatoid arthritis. SNHL was reported in $94.02 \%$ patients in right ear and $95.73 \%$ in left ear for a frequency range of $10,000 \mathrm{~Hz}$ to $16,000 \mathrm{~Hz}$. Dissolution of disk material at the synovial joint, proliferation and formation of extra articular tissues at the joint surfaces can be reasoned to the greater hearing thresholds at higher frequencies [15].

Similar to other autoimmune diseases, RA may also cause SNHL $[11,12,14,23]$. The etiology for SNHL in RA has been a subject of debate. In the present study, 37 subjects (92.5\%) had SN type hearing loss in both ears. Some authors attribute the SN component to vasculitis or neuritis [15]; arteritis of the vasa nervorum of the auditory nerve [24-29]. The other cause for SN hearing loss could be drug induced ototoxicity. Heyworth et al. [23] reported that salicylate intake did not cause hearing loss and the same was confirmed in cases having hearing loss without any history of salicylate intake. However, in the present study, the effect of medication/drug otoxicity was not taken into consideration.

In the present study, majority of the subjects (90\%) had normal tympanometric findings. The middle ear pressure and static compliance were within normal limits for all subjects, except for Type B tympanogram in three cases. Treviño et al. [30] have also reported Type A tympanograms using impedance audiometry for $88.90 \%$ and $91.46 \%$ subjects in right and left ears respectively. Contrary to the present findings, authors have reported abnormal tympanometric findings for the RA group with the majority of subjects having stiffness related pathology or ossicular chain discontinuity $[13,24,25,27]$. The use of a 226 $\mathrm{Hz}$ probe tone for impedance measurement can be the reason for disagreement with other studies. Whereas, most of the studies in the literature have evaluated middle ear function using multi frequency Tympanometry.

Stapedial reflex test was done to assess the stapedial muscle contraction reflex to high intensity stimulus. The findings in the present study suggest an absence of ipsilateral acoustic reflexes in $31(77.5 \%)$ and $3(7.5 \%)$ subjects of the RA and control groups respectively. Contralateral reflexes were absent for 35 (87.5\%) and $3(7.5 \%)$ subjects in the RA and control group respectively. The absence of acoustic reflexes could possibly be related to reduced sound energy reaching the cochlea, due to the presence of hearing loss. Hüttenbrink KB [31] has also reported similar findings and hypothesised that when pressure changes are induced, an altered functioning of the anvil and hammer in the ossicular chain leads to reduced protective mechanism of the middle ear muscles to elicit reflex.

The integrity of the cochlea was assessed using the OAE system. TEOAE's were compared between the two groups. An absence/ reduction of TEOAE in $72.5 \%$ subjects in right ear and $67.5 \%$ subjects in left ear were suggestive of a hair cell dysfunction in the RA group. An inner hair cell injury has been hypothesised as a probable site of lesion resulting in hearing loss in RA population [22]. Hearing loss in RA has also been correlated with disease progression. Öztürk et al. [15] reported increased hearing thresholds with SN hearing loss and reduced amplitude for TEOAE during the early stages of the disease. Salvinelli et al. [22] have suggested an inverse correlation between duration of disease and TEOAE amplitude. Their results suggest TEOAE as an easy and non invasive technique to stage disease progression.

Auditory Brainstem responses were recorded for both the groups using standard measurement protocol for adults at 90 $\mathrm{dB} n \mathrm{~nL}$ and $70 \mathrm{~dB}$ nHL. The results of the present study revealed a I-III interpeak latency prolongation in $7(17.5 \%)$ and $4(10 \%)$ subjects for right and left ears respectively. A prolongation in $\mathrm{I}-\mathrm{V}$ interpeak latency was also obtained for $3(7.5 \%)$ and $2(5 \%)$ subjects with RA in the right and left ear respectively, though a statistically significant difference was not obtained between the two groups. The results of the present study suggest that the site of lesion may also involve the lower brainstem.

Though there is limited research documenting the effect of RA on the functioning of the central auditory pathway, few authors have reported prolongation of wave I, III and V and IPL delay at high repetition rates for the RA group [27]. Murdin et al. [28] hypothesised that abnormal ABR findings with absent acoustic reflexes are suggestive of retrocochlear pathology. The present study also reported a correlation between the Rheumatoid Factor (RF) and the audiological parameters suggesting that an increase in disease progression positively correlated with increase in hearing threshold. A direct relationship between the admittance audiometry, RA factor, staging and activity of the disease with its progression has been reported [29]. However, the staging and disease activity was not taken into account in the present study. 


\section{Global Journal of Otolaryngology}

\section{Conclusion}

The results of the present study indicate that extra articular manifestations in RA are hearing loss and middle ear muscle reflex abnormality. The central auditory mechanisms can also be affected as revealed by the abnormal ABR findings. Hence, both the peripheral and central auditory mechanisms can be altered in RA. Although these results are in coherence with other research findings, the study also presents with certain limitations: Firstly, the effect of disease progression has not been monitored for the study group. Secondly, limited tests have been administered to study retro cochlear involvement in RA. Future research should be directed on studying the effect of disease progression on auditory evoked potentials to identify the site of lesion along the auditory pathway.

\section{Conflict of interest}

The authors hereby report no conflict of interest from any possible sources. The authors also hereby disclose that no external grants or sponsorship were received for the research study.

\section{References}

1. Baradaranfar MH, Doosti A (2010) A survey of relationship between rheumatoid arthritis and hearing disorders. Acta Med Iran 48(6): 371373.

2. Broker DS (1988) Rheumatoid arthritis: otorhinolaryngological manifestations. Clin Otolaryngol Allied Sci 13(3): 239-246.

3. Kasper DL, Brounwald E, Fauci AS, Hauser SL, Longo DL, et al. (2005) Harrison's Principles of Internal Medicine. (16 ${ }^{\text {th }}$ edn), McGraw-Hill, New York, USA, pp. 1968-1977.

4. Gairola A, Kacker S, Kumar A, Malaviya A (1991) Laryngeal and ear involvement in rheumatoid arthritis in north India. $\mathrm{Br} \mathrm{J}$ Rheumatol 30(1): 65-66.

5. Harris ED (2005) Prednisolone in early rheumatoid arthritis: an anti invasive effect. Arthritis Rheum 52(11): 3324-3325.

6. Kovarsky J (1984) Hydroxychloroquine in rheumatoid arthritis Arthritis Rheum 27(11): 1315-1316.

7. Brazeau-Lamontagne L, Charlin B, Levesque RY, Lussier A (1986) Cricoarytenoiditis: CT assessment in rheumatoid arthritis. Radiology 158(2): 463-466.

8. Kolman J, Morris I (2002) Cricoarytenoid arthritis: a cause of acute upper airway obstruction in arthritis. Can J Anaesth 49(7): 729-732.

9. Papadimitraki ED, Kyrmizakis DE, Kritikos I, Boumpas DT (2004) Earnose-throat manifestations of autoimmune rheumatic diseases. Clin Exp Rheumatol 22(4): 485-494.

10. Voulgari PV, Papazisi D, Bai M, Zagorianakou P, Assimakopoulos D, et al. (2005) Laryngeal involvement in rheumatoid arthritis. Rheumatol Int 25(5): 321-325.

11. Magaro M, Zoli A, Altomonte L, Mirone L, Corvino G, et al. (1990) Sensorineural hearing loss in rheumatoid arthritis. Clin Exp Rheumatol 8(5): 487-490.

12. Kastanioudakis I, Skevas A, Danielidis V, Tsiakou E, Drosos AA, et al. (1995) Inner ear involvement in rheumatoid arthritis: a prospective clinical study. J Laryngol Otol 109(8): 713-718.

13. Ozcan M, Karakuş MF, Gündüz OH, Tuncel U, Sahin H (2002) Hearing loss and middle ear involvement in rheumatoid arthritis. Rheumatology International 22(1): 16-19.

14. Takatsu M, Higaki M, Kinoshita H, Mizushima Y, Koizuka I.(2005) Ear involvement in patients with rheumatoid arthritis. Otol Neurotol. Jul;26(4):755-61.

15. Oztürk A, Yalçin S, Kaygusuz I, Sahin S, Gök U, et al. (2004) Highfrequency hearing loss and middle ear involvement in rheumatoid arthritis. American Journal of Otolaryngology 25(6): 411-417.

16. Walek H, Fritze W, Kolarz G (1980) Possible involvement of the auditory system in rheumatoid arthritis. Z Rheumatol 39(3-4): 91-94.

17. Raut VV, Cullen J, Cathers G (2001) Hearing loss in rheumatoid arthritis. J Otolaryngol 30(5): 289-294.

18. Alonso Laura, Ileana Gutierrez-Farfan, Angelica Pẽna-Ayala, MariaEsther Perez-Bastidas, et al. (2011) Clinical Significance of Auditive Involvement in Rheumatoid Arthritis: A Case-Control Study. ISRN Rheumatology 2011(2011): 7.

19. Clark JG (1981) Uses and abuses of hearing loss classification. ASHA 23(7): 493-500.

20. Jerger J (1970) Clinical experience with impedance audiometry. Arch Otolaryngol 92(4):311-324

21. Salvinelli F, D’Ascanio L, Casale M, Vadacca M, Rigon A, et al. (2006) Auditory pathway in rheumatoid arthritis. A comparative study and surgical perspectives. Acta Otolaryngol 126(1): 32-36.

22. Salvinelli F, D’Ascanio, Casale M (2004) Staging Rheumatoid Arthiritis: What about Oto Acoustic Emissions? Acta l Otolaryngol 124(7): 874887.

23. Heyworth T, Liyanage SP (1972) A Pilot Survey of Hearing Loss in Patients with Rheumatoid Arthritis. Scandinavian Journal Of Rheumatology 1(2): 81-83.

24. Goodwill CJ, Lord IJ, Jones RP (1972) Hearing in rheumatoid arthritis: A clinical and audiometric survey. Annals of the Rheumatic Diseases 31(3): 170-173.

25. Colletti V, Fiorino FG, Bruni L, Biasi D (1997) Middle Ear Mechanics in Subjects with Rheumatoid Arthritis. Audiology 36(3): 136-146.

26. Djupesland G, Gronås HE, Saxegaard EF (1973) Hearing and Middle-Ear Function In Patients With Inflammatory Rheumatoid Joint Diseases. Scand J Rheumatol 2(2): 53-56.

27.Zahraa I Selim, Sherifa A Hamed, Amal M Elattar (2015) Peripheral and Central auditory Pathway function in rheumatoid arthritis. International Journal of Clinical Rheumatology 10(2): 85-96.

28. Murdin L, Patel S, Walmsley J, Yeoh LH (2008) Hearing difficulties are common in patients with rheumatoid arthritis. Clin Rheumatol 27(5): 637-640.

29. Poorey VK, Khatri R. (2001) Study of auditory function in rheumatoid arthritis. Indian J Otolaryngol Head Neck Surg 53(4): 261-263.

30. Treviño-González JL, Villegas-González MJ, Muñoz-Maldonado GE, Montero-Cantu CA, Nava-Zavala AH, et al. (2015) Subclinical sensorineural hearing loss in female patients with rheumatoid arthritis. Cir Cir 83(5): 364-370.

31. Hüttenbrink KB (1987) Scanning electron microscopy studies in arthritic changes in the malleus incus joint and reflections on the function of the middle ear muscles. Laryngol Rhinol Otol (Stuttg) 66(4): 180-185.

32. Dikici O, Muluk NB, Tosun AK (2009) fe Sensorineural hearing loss in female patients with rheumatoid arthiritis. Eur Arch Otorhinolaryngol 266: 1719. 
Your next submission with JuniperPublishers will reach you the below assets

- Quality Editorial service

- Swift Peer Review

- Reprints availability

- E-prints Service

- Manuscript Podcast for convenient understanding

- Global attainment for your research

- Manuscript accessibility in different formats

( Pdf, E-pub, Full Text, Audio)

- Unceasing customer service 